\title{
Validación del material lúdico de la estrategia educativa basada en juegos para la promoción de estilos de vida saludable en niños de cuatro a cinco años de edad
}

\author{
Martha Liévano-Fiesco*, Gloria García-Londoño, Martha Leclercq-Barriga, \\ Gladys Liévano-De Lombo, Karen Solano-Salazar \\ Departamento de Nutrición y Bioquímica. Facultad de Ciencias. \\ Pontificia Universidad Javeriana. Cra. $7^{a}$ No. 43-82. Bogotá, Colombia \\ *mlievano@javeriana.edu.co
}

Recibido: 08-04-2008; Aceptado: 15-05-2009

\begin{abstract}
Resumen
Objetivo: validar los juegos diseñados para desarrollar las temáticas de la estrategia pedagógica lúdica en alimentación y nutrición, del programa "Paseando por el Mundo Mágico de una Alimentación Saludable" como método de enseñanza-aprendizaje, para la promoción de estilos de vida saludable, en población preescolar de 4 a 5 años; y, determinar el logro de los objetivos de aprendizaje propuestos. Materiales y métodos: Esta investigación se realizó en dos etapas: la primera etapa comprendió el desarrollo de las temáticas con 37 niños del curso Jardín A, mediante la utilización de los seis juegos. Para la evaluación del aprendizaje significativo se realizó una evaluación antes y después de desarrollar el programa. El análisis de los resultados se hizo mediante una prueba t pareada. La segunda etapa comprendió la validación los juegos: Concéntrese "Busca la pareja y ganarás"; Rompecabezas "Armando el tren de la Alimentación Saludable"; Cartas "Nutri-cartas"; Bingo "Nutri-salud"; Lotería "Nutriderechos” y Escalera "Lleguemos al mundo mágico de la alimentación”, para lo cual se seleccionaron 12 niños, 4 padres de familia, 4 personas de la institución (3 profesores y la directora) y 4 expertos profesionales nutricionistas dietistas en Bogotá D.C. Resultados: El promedio de edad de los niños fue de 4,6 0,31 años. Antes de la intervención educativa, la calificación promedio fue de 27 puntos sobre 50 y después de 36,1 (p<0,001). Las variables estudiadas para la validación fueron: atractividad, entendimiento, identificación de acciones cotidianas, aceptación e inducción a la acción. Conclusión: En general tanto los niños como los adultos, aceptaron los juegos e hicieron aportes para mejorarlo.
\end{abstract}

Palabras clave: alimentación saludable, educación alimentaria y nutricional, estilos de vida saludables, material educativo, material lúdico, juegos, promoción de la salud, validación.

\begin{abstract}
Validation of the ludic material of the educational strategy based on games for the promotion of healthy lifestyles in children of four to five years old. Objectives: (1) to validate the games designed to develop the thematic contents of the ludic pedagogical strategy for diet and nutrition of the program "Walking through the Magical World of Healthy Eating" as a learning-teaching method, for the promotion of healthy lifestyles in a preschool population with ages from 4 to 5; and (2) to determine the fulfillment of the expected learning outcomes by the children. Materials and methods: This investigation was developed in two phases: the first phase comprised the development of the thematic contents with 37 children of the course Nursery A by using six games. For the evaluation of significant learning, an evaluation was carried out before and after developing the program. The analysis of results was done by a paired t-test. The second phase comprised the validation the following games: "Find the missing pair and win" (concentrate); "Building the train of Healthy Diet" (puzzle); "Nutri-Cards"; "Nutri-Health" (bingo); "Nutri-rights" (lottery) and "Let's arrive to the magic world of the diet" (stairs). For this purpose we selected 12 children, 4 parents, 4 people of the institution (3 teachers and the Head Master) and 4 professional nutritionist-dieticians in Bogotá D.C. Results: The average age was $4.6 \pm 0.31$ years old. Before the educational intervention, the average score was of 27 points above 50 and after the educational intervention it was of 36.1 points $(\mathrm{p}<0.001)$. The variables studied for the validation were: attractiveness, understanding, identification of daily actions, acceptance and induction to the action. Conclusion: In general, both children and adults accepted the games and contributed for their improvement.
\end{abstract}

Key words: dietary and nutritional education, educational material, games, health promotion, healthy diet, healthy lifestyles, ludic material, validation. 


\begin{abstract}
Resumo
Validação do material lúdico da estratégia educativa baseada em jogos para promoção de estilos de vida saudáveis em crianças de quatro a cinco anos de idade. Objetivo: validar os jogos feitos para desenvolver as temáticas da estratégia pedagógica lúdica na alimentação e nutrição, do programa "Passeio pelo Mundo Mágico de uma Alimentação Saudável" como método de ensino-aprendizagem, para o incentivo de estilos de vida saudáveis, na população pré-escolar de 4 a 5 anos; e, determinar o sucesso dos objetivos de aprendizagem propostos. Materiais e Métodos: Esta pesquisa realizou-se em duas etapas: a primeira abrange o desenvolvimento das temáticas com 37 crianças do curso Jardim A, utilizando seis jogos. Para a avaliação da aprendizagem significativa realizou-se uma avaliação antes e depois de aplicar o programa. A análise dos resultados realizou-se com uma prova t pareada. A segunda etapa validou os jogos: Memória "Encontra o par e ganhará"; Quebra-cabeças "arma o trem da Alimentação Saudável”; Baralho "Nutre-baralho"; Bingo "Nutre-saúde"; Loteria "Nutre - direitos" e Escada "Entremos ao mundo mágico da alimentação"; para isto, selecionaram-se 12 crianças, 4 pais de família, 4 pessoas da instituição (3 professores e a diretora) e 4 expertos profissionais nutricionistas dietistas em Bogotá, D.C. Resultados: A média da idade das crianças foi de 4,6 0 0,31 anos. Antes da intervenção pedagógica, a qualificação média foi de 27 pontos sob 50 e depois de 36, 1 (p<0,001). As variáveis estudadas para a validação foram: atratividade, entendimento, identificação de ações cotidianas, aceitação e induç ão à ação. Conclusão: No geral, crianças e adultos aceitaram os jogos e fizeram aportes para melhorar-los.
\end{abstract}

Palavras chave: alimentação saudável, educação alimentícia e nutricional, estilos de vida saudáveis, material educativo, material lúdico, jogos, promoção da saúde, validação.

\section{Introducción}

Teniendo en cuenta que los hábitos, prácticas, conocimientos y actitudes alimentarias de una población constituyen un factor decisivo en su nivel de salud, relacionándose directa o indirectamente con enfermedades de alta prevalencia y mortalidad, se plantea la necesidad de desarrollar procesos de educación en alimentación y nutrición, que conlleven a unos comportamientos favorables para la salud de la población en especial de los niños, etapa en la cual se adquieren las actitudes y patrones de conducta que regulan la vida en la edad adulta. Para esto, se debe tener en cuenta que los materiales educativos que se utilicen sean adecuados, con el fin de asegurar mejores niveles de entendimiento, identificación y percepción entre otros aspectos, por lo que, se hace indispensable llevar a cabo procesos de validación del material educativo a utilizar $(1,2)$.

La validación de materiales educativos, definida como la investigación que se realiza con el fin de que representantes de un grupo de personas a los cuales va dirigido un material específico (población meta, grupo objetivo), opinen sobre los instrumentos o materiales que se han elaborado, antes que éstos ingresen a la etapa final o de manufactura de los mismos $(3,4)$, busca comprender las particularidades de un grupo objetivo, por ser ellos mismos quienes señalan sus propias características. Sin validación, muchas intervenciones con materiales educativos dificultarían determinar el logro del objetivo propuesto.

Con el desarrollo de este proyecto, se pretende que la validación se configure como un proceso científico, de conocimiento y comprensión para la comunicación, fundamental para que no se continúe con una incomunicación y separación entre la población a intervenir y las instituciones que presentan los programas educativos, que contribuyen, en la mayoría de los casos, a la formación y/o fortalecimiento de conocimientos y prácticas alimentarias.

El propósito principal del presente estudio es validar los juegos diseñados en estudios anteriores como método de enseñanza-aprendizaje para la promoción de estilos de vida saludable en población preescolar de 4 a 5 años del Jardín Infantil Sol Solecito, de la localidad de Suba en la ciudad de Bogotá D.C. Además, determinar si después del desarrollo de las temáticas que hacen parte de la estrategia educativa lúdica, se lograron aprendizajes significativos en los niños.

\section{Materiales y métodos}

La investigación se desarrolló en dos etapas consecutivas: la primera etapa comprendió el desarrollo de las temáticas de la estrategia pedagógica lúdicas, utilizando para ello los juegos que hacen parte del programa educativo " $\mathrm{Pa}$ seando por el Mundo Mágico de una Alimentación Saludable", diseñado en investigaciones anteriores por las autoras, y la segunda etapa estuvo centrada en la validación del material educativo (juegos) con niños, padres de familia, profesores de la institución educativa; y expertos profesionales nutricionistas dietistas en Bogotá D.C., Colombia.

Para la primera etapa, la muestra estuvo conformada por 37 niños de cuatro a cinco años de edad del grado Jardín A, pertenecientes al Jardín infantil Sol Solecito de la localidad de Suba, Bogotá. En condiciones basales se aplicó un cuestionario estandarizado para evaluar los conocimientos y las actitudes de los niños sobre conceptos básicos en 
alimentación y nutrición, en las áreas de hábitos alimentarios, hábitos higiénicos, actividad física y derechos de los niños. Una vez se desarrollaron las temáticas en alimentación y nutrición utilizando estrategias metodológicas lúdicas, se aplicó nuevamente el cuestionario para evaluar el logro de los objetivos de aprendizaje propuestos en el programa educativo. Para determinar si existía o no diferencia entre los conocimientos basales y finales, se realizó una comparación por parejas utilizando la prueba $t$-student.

Posteriormente, y de manera aleatoria para la segunda etapa de validación, se escogieron 12 niños del grado Jardín A y, por muestreo no probabilístico y por conveniencia, se seleccionaron 4 de padres de familia, 4 docentes y 4 expertos profesionales nutricionistas dietistas con experiencia en diferentes campos de desempeño en el área de nutrición de Bogotá D.C., Colombia.

Las variables del estudio fueron: atracción, entendimiento, identificación, aceptación e inducción a la acción; las cuales se clasificaron de acuerdo a las recomendaciones de la literatura (3), y adaptadas para el propósito del proyecto.

La recolección de la información se realizó mediante entrevista personal, diligenciando la ficha de validación diseñada para cada juego y para cada grupo validador (niños y adultos). La tabulación y análisis se hizo utilizando el programa Epi Info 3.3.2.

\section{Resultados}

\section{Evaluación del aprendizaje}

El promedio de edad de los niños fue de 4,6 \pm 0,31 años con mayor participación del género femenino con un $68 \%$.

El cuestionario para evaluar los conocimientos basales y finales se le aplicó a 37 niños del grado Jardín A. El promedio obtenido por el grupo de niños antes de la intervención educativa fue de 26,8 puntos sobre 50 , y el promedio después fue de 41,2. Al aplicar la prueba $t$ se observó que con la intervención educativa utilizando estrategias metodológicas lúdicas, se logran los objetivos de aprendizaje propuestos en el programa. $(\mathrm{p}<0,05)$.

\section{Validación del material educativo}

Se validaron seis juegos:

- Concéntrese "Busca la pareja y ganarás"
Tema: Origen de los alimentos.

Objetivo: Al término del juego concéntrese "busca la pareja y ganarás", los niños estarán en capacidad de identificar a qué reino de la naturaleza pertenecen los alimentos que hacen parte de la alimentación diaria.

- Rompecabezas "Armando el tren de la alimentación saludable"

Tema: Conozcamos nuestros amigos los alimentos.

Objetivo: Al terminar el rompecabezas "Armando el tren de la alimentación saludable", los niños estarán en capacidad de identificar los alimentos que se encuentran en cada vagón del tren, así como su función y la importancia de incluirlos diariamente en su alimentación, para un adecuado crecimiento y desarrollo.

- Cartas "Nutri-cartas".

Tema: Funciones de los alimentos.

Objetivo: Al término del juego de cartas "Nutri-cartas", los niños estarán en capacidad de identificar la función que cumplen los alimentos pertenecientes a cada uno de los tres reinos, para un adecuado crecimiento y desarrollo.

- Bingo "Nutri-salud".

Temas: higiene personal y de los alimentos y actividad física.

Objetivo: Al término del bingo "Nutri-salud", los niños estarán en capacidad de identificar las normas de higiene personal y de los alimentos, como parte de su rutina diaria para prevenir enfermedades.

Enumerar las ventajas de practicar actividad física diariamente para mantener un estado de salud adecuado.

- Lotería "Nutri-derechos"

Tema: Los derechos del niño.

Objetivo: Al término del juego con la lotería "Nutriderechos", los niños estarán en capacidad de identificar sus derechos como niños.

- Escalera "Lleguemos al mundo mágico de la alimentación”.

Objetivo: Al término del juego de escalera "Lleguemos a Nutrilandia", los niños estarán en capacidad de:

* Identificar los alimentos y su origen.

* Identificar los alimentos que deben incluir diariamente en su alimentación para un adecuado crecimiento y desarrollo.

* Enunciar las reglas de higiene personal y de los alimentos como parte de su rutina diaria para prevenir enfermedades. 
* Enunciar las ventajas de la actividad física diaria para mantener un estado de salud adecuado.

* Identificar sus derechos como niños.

Obteniéndose los siguientes resultados para cada una de las variables estudiadas:

\section{Atracción}

Se evaluó contemplando los siguientes aspectos: colores, tamaño del juego, tamaño de los dibujos, dibujos que más y que menos gustaban en cada uno de los juegos, motivación para continuar el juego, así como el juego de mayor y menor preferencia.

En los niños, se observó que los colores más llamativos fueron los colores primarios, con un 58,3\% para el concéntrese y $41,7 \%$ para la lotería, para la escalera el color de mayor atracción fue el rojo, para el bingo fue el azul $(66,7 \%)$, para el rompecabezas fue el verde, mientras que en las cartas no hubo un color predilecto. Los adultos, consideraron los colores de todos los juegos adecuados porque "Conservan su color natural tal como el niño o la niña los conoce", además "son colores que despiertan el interés y motivan a los asistentes a prestar atención”.
En relación con el tamaño de los juegos, la mayoría de los niños consideraron que era el ideal para el concéntrese $(66,7 \%)$, las cartas $(58,3 \%)$, el bingo $(50 \%)$ y la lotería $(50 \%)$, mientras que para el rompecabezas $(58,3 \%)$ y la escalera $(75 \%)$ consideraron que debe ser más grande (Ver figura 1). El $50 \%$ de los padres y de los nutricionistas opinaron, que el tamaño del cartón de la escalera debe ser más grande.

La opinión de los tres grupos de adultos relacionada con cuál de los juegos es el más apropiado para el grupo de edad, fue para el personal institucional en su mayoría consideraron que todos los juegos son adecuados, mientras que el $75 \%$ de los padres de familia y los nutricionistas piensan que las cartas y el concéntrese no son adecuados.

Al evaluar cuál de los juegos les gusto más y cuál menos, el $41,7 \%$ de los niños mencionó que todos los juegos les había gustado por igual, el 33,3\% prefirieron el concéntrese y el bingo, y el juego que menos gustó fue la lotería con un $25 \%$ (ver Figura 2).

\section{Entendimiento}

Este aspecto fue evaluado por medio de preguntas relacionadas con la metodología para desarrollar el juego (ins-

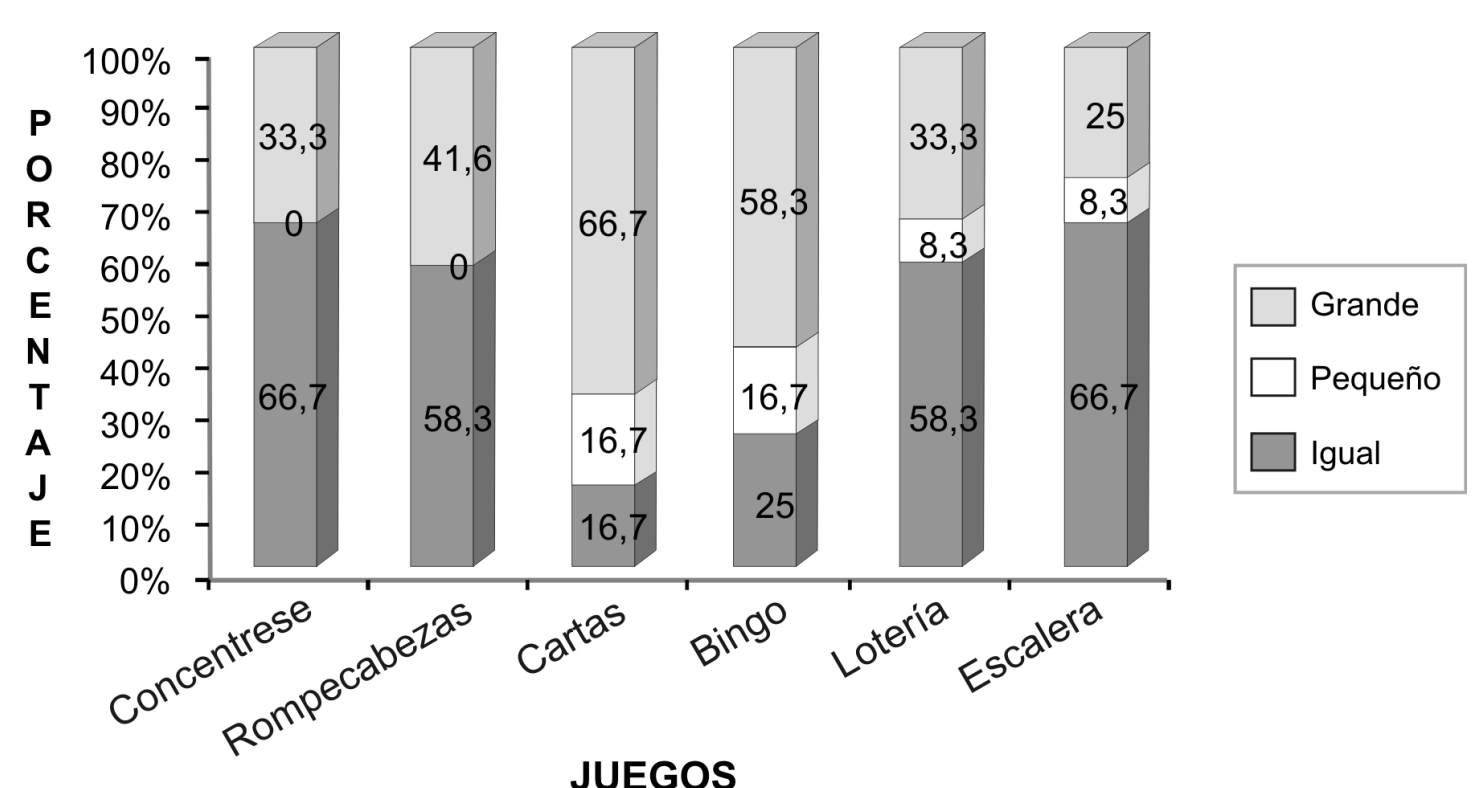

Figura 1. Tamaño del cartón del juego. Niño(a)s de 4 a 5 años curso Jardín A. Pertenecientes al Jardín Infantil Sol Solecito localidad de Suba. Bogotá, D.C. $(\mathrm{N}=12)$ 


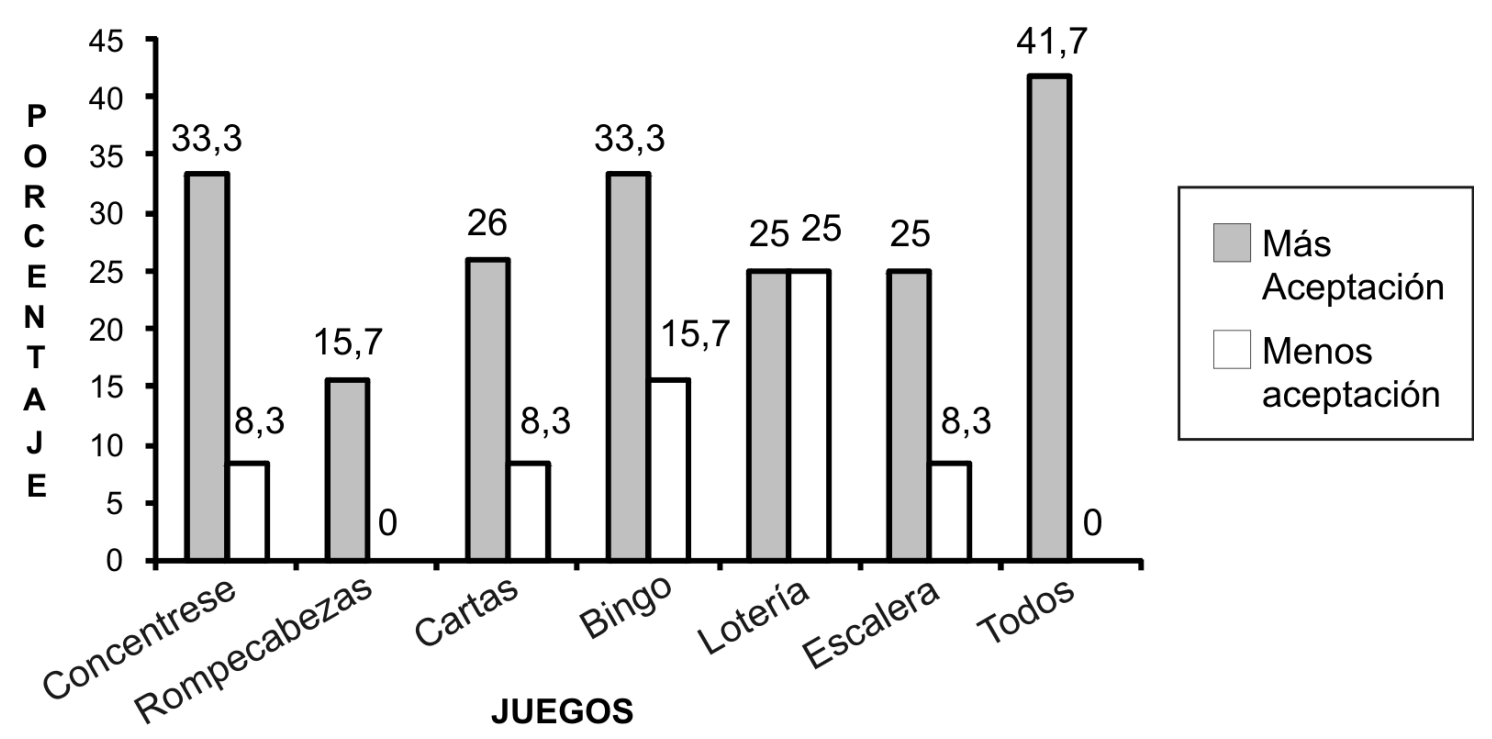

Figura 2. Distribución porcentual de acuerdo a la aceptación de cada uno de los juegos. Niños(as) de 4 a 5 años curso Jardín A, pertenecientes al Jardín Infantil Sol Solecito localidad de Suba. Bogotá, D.C. (N=12)

tructivo) así como, en la identificación de figuras y lo que representa cada una de ellas.

Tanto los niños como los tres grupos de adultos coincidieron en que el instructivo era adecuado.

En relación con los dibujos identificados correctamente para cada uno de los juegos se encontró:

Concéntrese: el 100\% de los niños los identificaron correctamente la piña, el agua, los bananos y el huevo, por el contrario la carne sólo el 8,3\% la identificó correctamente, seguida por las lentejas con un $25 \%$.

Rompecabezas, el dibujo que la mayoría $(91,7 \%)$ de los niños identificó correctamente fue el vagón de las frutas.

Cartas, alrededor del $60 \%$ de los niños identificaron todos los alimentos, el que mejor identificaron fue el pan (75\%).

Bingo, el papel higiénico, el juego de bolos y el lavado de manos fueron las imágenes que en mayor proporción fueron identificadas correctamente por los niños, llama la atención que en este juego ningún niño identificó correctamente las raquetas.

Lotería, las imágenes que representaban los derechos a la alimentación, la comprensión y a la familia fueron identificados correctamente por el $100 \%$ de los niños.
Escalera, en su mayoría las imágenes fueron identificadas, pero sólo el $25 \%$ de los niños identificó correctamente la imagen relacionada con el lavado de manos.

\section{Identificación}

Este aspecto fue evaluado en los niños con la identificación de acciones o elementos cotidianos o del medio de convivencia del menor con el juego. Se encontró que los alimentos más conocidos en la tienda, plaza o almacén, para cada uno de los juegos son: el tomate (100\%) en el concéntrese, el vagón de las frutas $(91,7 \%)$ en el rompecabezas, la zanahoria, la piña y el tomate $(100 \%)$ en las cartas y en la escalera, la zanahoria $(91,7 \%)$ y en la casa los alimentos más conocidos por el $100 \%$ de los niños fueron la piña, el tomate, el agua, la carne, los bananos, los lácteos y el huevo.

Con la lotería, los resultados de identificación de los derechos del niño, en el hogar en primer lugar se ubicó el derecho a la salud con el $91,7 \%$ seguido con un $83,3 \%$ el derecho a la alimentación, educación, comprensión, no abandono, al juego y a la libertad de expresión. Sin embargo, los derechos más identificados por los niños en el jardín fueron el derecho al juego y a la libertad de expresión con un $91,7 \%$.

En cuanto a la acción más identificada por ellos en el juego de las cartas fue un niño en el médico con el 100\%, para 
el bingo la acción de mayor identificación es el lavado de manos con un $100 \%$, en el caso de la lotería los derechos más identificados por los niños como acciones que practican fueron el derecho a la alimentación, al amor, a la comprensión y a la libertad de expresión con un $100 \%$.

En los adultos, fue evaluado como la identificación de los niños con el juego, si el tema es adecuado para el grupo de edad, y si cumple con la cotidianidad del niño. Para la pregunta si creen que los niños se sentirán identificados con el juego los tres grupos opinaron que sí, en el bingo, lotería y escalera, en el concéntrese y cartas y rompecabezas el $100 \%$ de los padres y personal institucional estuvieron de acuerdo frente a un $75 \%$ del grupo de nutricionistas. Para el tema tratado, el $100 \%$ de cada uno de los grupos estuvo de acuerdo en que el tema en cada uno de los juegos es adecuado para este grupo de edad, excepto para la lotería donde solamente el $75 \%$ consideraron que sí lo era y opinaron que la mayoría de los juegos son temas de la cotidianidad del niño.

\section{Aceptación}

En este aspecto se quería evaluar cuál era la aceptación del niño con el juego, para lo cual se les preguntó si les gustaría volver a jugarlo, el 97,2\% de los niños respondieron que sí y el 93,1\% de los niños jugarían con sus amigos los seis juegos.

Para los adultos, se evaluaron aspectos, sobre la aceptación del niño por los juegos, si los juegos contenían alguna idea que no fuera aceptada, y cuál figura, palabra o acción se debería cambiar. El 100\% de los tres grupos de adultos consideran que los niños aceptaran los juegos concéntrese, bingo y escalera. En relación con la lotería el $100 \%$ de los nutricionistas y padres y el 50\% del personal institucional consideran que los niños la aceptaran. Para la pregunta relacionada con si alguna idea contenida en los juegos no es aceptada por el niño, expresaron afirmativamente, debido a que algunas figuras no son claras y por lo tanto sugieren cambiarlas.

\section{Inducción a la acción}

En este aspecto se evaluaron los conocimientos y la motivación a nuevos comportamientos. Se observó que para cada juego los niños tenían claro cuál había sido el tema visto. Para las preguntas ¿por qué debemos consumir diariamente alimentos de todos los grupos del tren?, para crecer mucho fue la respuesta más común con el 41,7\%, ¿por qué tenemos que consumir una alimentación saludable?
Nos hace crecer sano y fuerte y los huesos fuertes y no se rompan contestaron con el 33,3\% y ¿por qué debemos hacer ejercicio diariamente? Para ser fuerte con el $50 \%$.

Para los adultos, esta variable se identifica a través de las preguntas: después del juego los niños cambiarían algo en la alimentación, en la higiene o en la actividad física, en cuatro de los seis juegos (cartas, bingo, lotería y escalera) el $100 \%$ de los participantes están de acuerdo en que sí pueden generar modificaciones en la alimentación, la higiene y la actividad física de los niños. En los otros juegos los porcentajes estuvieron entre 75 y $100 \%$.

\section{Discusión}

Los resultados obtenidos en la primera parte de este estudio muestran que la utilización de juegos como estrategia metodológica en la promoción de estilos de vida saludable podría ser una herramienta útil para lograr un mayor aprendizaje en la población infantil. En este estudio se utilizaron seis juegos tradicionales con los cuales se logró los objetivos de aprendizaje en los niños que los jugaron $\mathrm{p}(<0,05)$. Es indudable que se requieren evaluaciones a mediano y largo plazo para determinar si los conocimientos adquiridos son perdurables y se ven reflejados en cambios de actitud y en mejores condiciones nutricionales y de salud, así como, si son necesarias actividades de refuerzo del aprendizaje, lo cual será motivo de estudios posteriores.

Lo anterior nos muestra, que debemos aprovechar la influencia que ejerce el juego en los niños para el logro de aprendizajes, pero uno de los aspectos que se debe tener en cuenta al realizar material educativo es su validación para que éstos tengan el efecto deseado $(4,5)$.

Estudios existentes como los realizados en la ciudad de Cienfuegos (Cuba), el programa interdisciplinario "yo quiero ser sano" (6), igual que el proyecto sobre Educación nutricional en las escuelas básicas estos realizado en Chile (7) y el realizado en el Brasil (8) con la validación de un material tecnológico han mostrado al igual que este estudio que para tener un mayor impacto en las intervenciones educativas que se realicen el material educativo debe ser validado, teniendo en cuenta los dos principios básicos de ésta: el primero, hay que tener presente que no se está evaluando al participante, sino al material; y el segundo, el mensaje sustancial no debe cambiarse y sea cual sea las respuestas de los participantes del estudio todas deben ser tomadas siempre en beneficio de corregir o mejorar el material educativo. 


\section{Conclusiones}

En general tanto los niños como el grupo de adultos, aceptaron los juegos, en cuanto a sus colores, figuras, materiales, temas tratados, acciones e identificación de imágenes; además dieron aportes importantes para mejorar el material.

Se observó además, que la utilización de los juegos, logró incrementar de manera significativa los conocimientos sobre alimentación y nutrición en los niño(a)s que los jugaron $(p<0,05)$, pero es necesario realizar evaluaciones a mediano y largo plazo para determinar si los conocimientos adquiridos son perdurables y son llevados a la práctica por parte de los niños.

En cuanto a la validación del material educativo, se evidencia la importancia de evaluar dichos materiales, ya que permiten enriquecerlos, de acuerdo a las opiniones de la misma población a la que van dirigidos. Estos aportes fueron soportados por las opiniones hechas por el grupo de validación de adultos y por la misma población a la que van dirigidos (niños).

\section{Agradecimientos}

Al Jardín Sol Solecito, por permitirnos el desarrollo de esta investigación, especialmente a la hermana María Isabel profesora Jardín A, por su colaboración y apoyo incondicional. Al Banco Arquidiocesano de Alimentos, por su apoyo económico, sembrando en investigación valiosa para la comunidad.

\section{Financiación}

La presente investigación hace parte del proyecto de investigación "Estrategias pedagógicas para la promoción de una alimentación saludable en población preescolar y escolar", presentado a la Convocatoria Interna año 2007, y se ejecutó con recursos de la Pontificia Universidad Javeriana

\section{Conflictos de intereses}

No se presentan.

\section{Referencias}

1. Haaland A. Validación de materiales de comunicación: con énfasis especial en la salud infantil y educación sobre nutrición. UNICEF. Guatemala. GT. 1990; 89 págs.

2. Medrano L, Núñez G. Manual de Validación de Materiales Educativos-Comunicacionales. Proyecto Salud y Nutrición Básica. Lima, Perú. 2001; 80 págs.

3. Ziemendorff S, Krause A. Guía de Validación de Materiales Educativos (con enfoque en materiales de educación sanitaria). Primera edición. Programa de Agua y Saneamiento PROAGUA/GTP. Chiclayo, Perú. 2003; 74 págs.

4. OPS/OMS. Orientaciones técnicas para el diseño y validación de materiales educativos para la iniciativa regional escuelas promotoras de la salud. Washington, D.C. 2005; 85 págs.

5. FAO. Validación del material educativo y de comunicación para la capacitación del personal y familias beneficiarias del programa de apoyo alimentario PalDiconsa. México. 2005; 42 págs.

6. Chiang M, Torres M, Maldonado M, y González U. Propuesta de un programa de promoción sobre un estilo de vida saludable en preescolares mediante una intervención multidisciplinaria. Revista cubana de investigaciones Biomédicas. 2003; 22 (4): 245-252.

7. Olivares S, Morón C, Zacarías I, Andrade M, Vio F. Educación en nutrición en las escuelas básicas de Chile. FAO. Instituto de Nutrición y Tecnología de los Alimentos (INTA). Universidad de Chile. Proyecto $\mathrm{TPC} / \mathrm{CHI} / 0065.2003 ; 18$ págs.

8. Martins V, Venicios M, Leite T. Validación de un recurso lúdico para la educación en salud cardiovascular. Universidad Federal de Ceará / Departamento de Enfermería. Brasil Revista Cubana de Enfermería. 2004; 20 (3): 236-240. 